\title{
Beaver tail liver in evans syndrome due to systemic lupus erythematosus
}

\section{KEYWORDS: SLE — beaver tail liver" evans syndrome" thrombocytopenia}

A 33 year old female diagnosed as Evans syndrome due to systemic lupus erythematosus (SLE), and lupus nephritis was further investigated for clinically enlarged liver and spleen [1]. The CT abdomen showed in addition to a mild hepato-splenomegaly, the presence of significant beaver tail liver (FIGURES 1 \&2). Though this incidental finding is of a benign nature it yet has some clinical implications particularly in a patient like this one, who has thrombocytopenia (platelets of $86.000 \mathrm{~mm}^{3}$ ), and hemorrhage is a potential complication. In case of trauma or injury to left side of the abdomen, which classically affects the spleen, this liver elongation may be affected and can be mistaken for splenic trauma or hemorrhage. Furthermore, the associations of this liver variant with other conditions are rather rare in the literature, so its coexistence here with a case of SLE related Evans syndrome has some academic orientation as well.

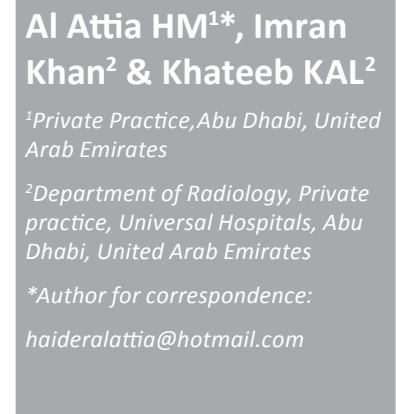

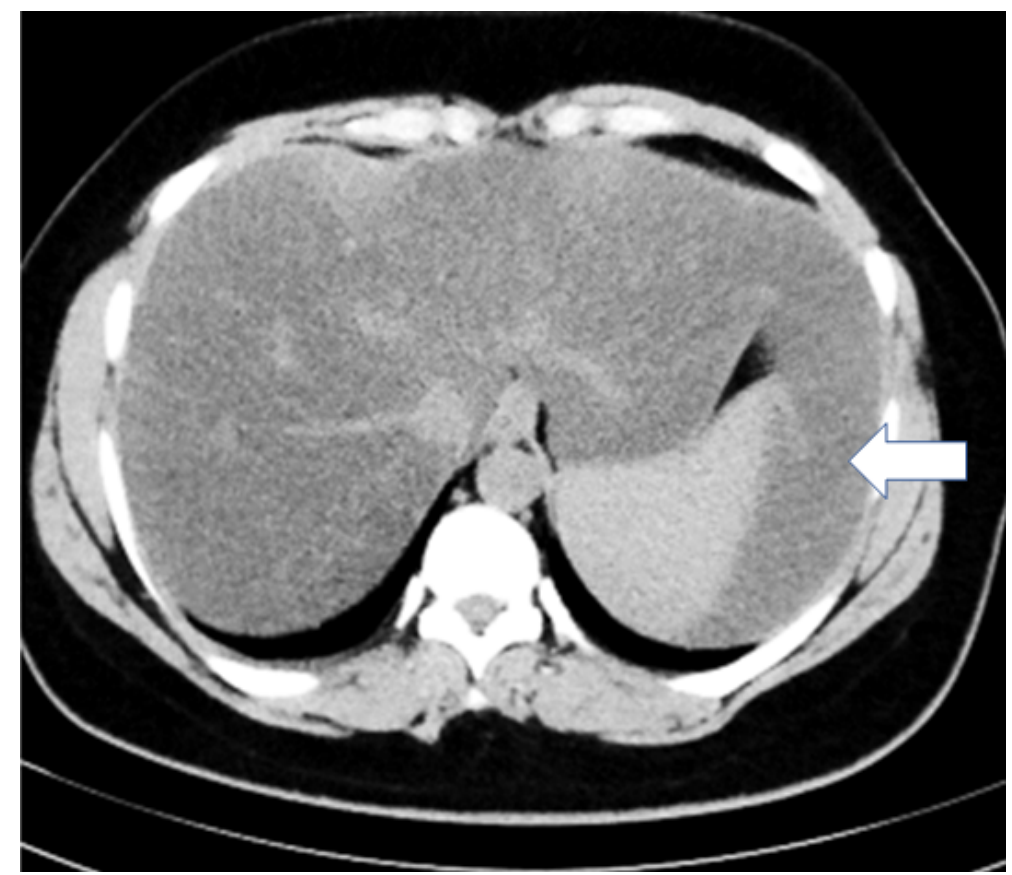

Figure 1. Abdominal CT scan (Axial view) showing beaver tail liver(arrow). It is extending beyond the spleen and surrounding it. 


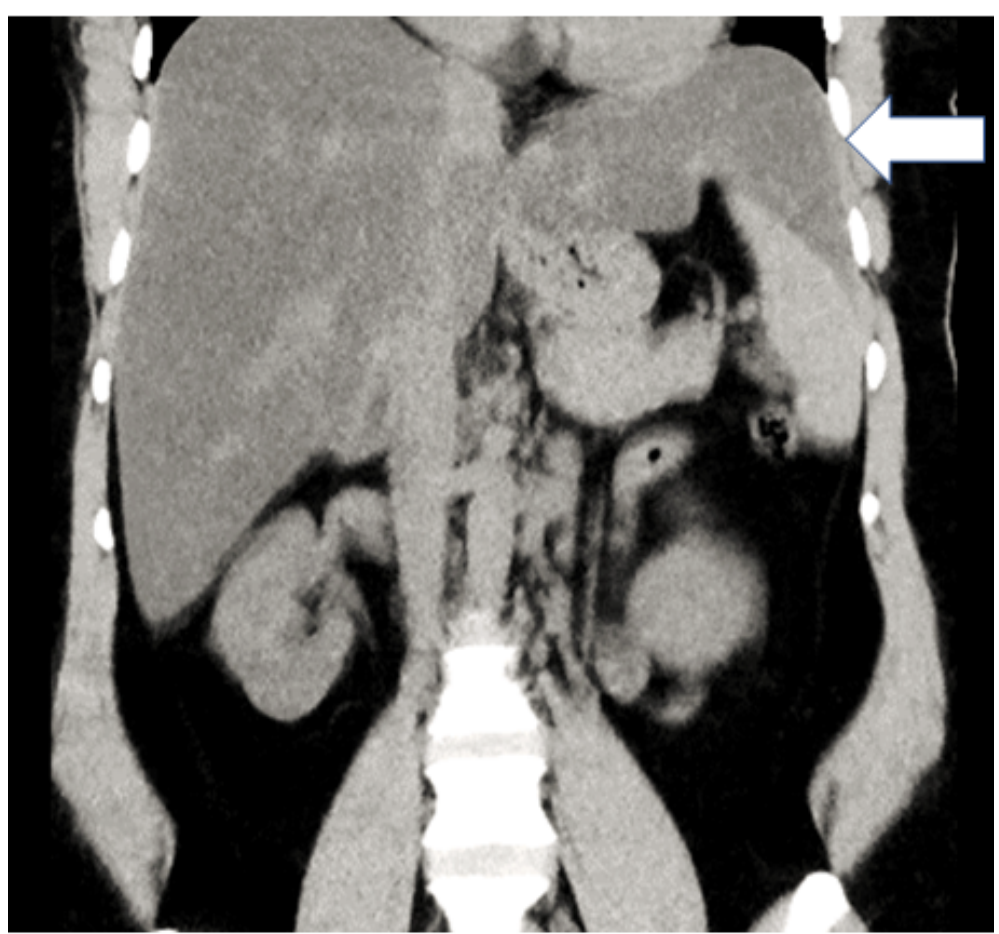

Figure 2. Abdominal CT scan (coronal view) showing the elongated liver (beaver tail) overlapping the spleen.

\section{REFERENCE}

1. Noh MSF, Jusna S, Muhammad SS. Beaver in the liver. Pan. Afr. Med. J. 27, 138 (2017). 